\title{
Mechanical Performance of Auxetic Polyurethane Foam for Antivibration Glove Applications
}

\author{
F. Scarpa*, J. Giacomin ${ }^{\circ}$, Y. Zhang*, and P. Pastorino* \\ *Multidomain Cellular Solids Laboratory, University of Sheffield, UK \\ ${ }^{\circ}$ Perception Enhancement Systems, University of Sheffield, UK
}

Received: 25 July 2005 Accepted: 2 September 2005

\begin{abstract}
In this study the static and dynamic characteristics of conventional open cell polyurethane (PU), of auxetic (negative Poisson's ratio) and of iso-density foams were analysed. The specimens were produced from conventional gray open-cells polyurethane foam with 30-35 pores/inch and $0.0027 \mathrm{~g} / \mathrm{cm}^{3}$ density, by means of process which has been previously defined by the authors. Poisson's ratio measurements were performed under quasi-static conditions using an MTS 858 servo-hydraulic test machine and a video image acquisition system. For the auxetic foams the results suggested similar behaviour to that previously reported in the literature, with significant increases in stiffness during compressive loading, and a significant dependence of the Poisson's ratio on the applied strain. Transmissibility tests, performed in accordance with the ISO 13753 procedure for antivibration glove materials, suggested a strong dependence of the transmissibility on the foam manufacturing parameters. Within the frequency range from 10 to $31.5 \mathrm{~Hz}$ the transmissibility was found to be greater than 1, while it was less than 1 at all frequencies greater than $31.5 \mathrm{~Hz}$. The transmissibility results were similar to the mean values for 80 resilient materials tested by Koton et. al., but were higher than the five best materials (not all polymeric) identified by the same researchers. In this study it has been suggested that the resilient behaviour of glove isolation materials should also be evaluated in terms of the indentation characteristics. A simple, linear elastic, Finite Element simulation was therefore performed, and the indentation results suggested that auxetic foams offer a significant decrease in compressive stresses with respect to conventional PU foams.
\end{abstract}

To whom correspondence should be addressed:

Dr Fabrizio Scarpa, Department of Aerospace Engineering, University of Bristol, BS8 1TR

Bristol, UK Tel: +44 1179289728 Fax: +44 1179773371 Email: f.scarpa@bris.ac.uk 


\section{INTRODUCTION}

With the entry into force in 2002 of the physical agents directive ${ }^{(1)}$, minimum requirements have been established in all European Union member states for the protection of workers against the possibly damaging effects of mechanical vibration. For hand-arm vibration, the physical agents directive establishes a daily exposure limit value of $5 \mathrm{~m} / \mathrm{s}^{2}$ of Wh weighted acceleration ${ }^{(2)}$, standardised to an eight-hour reference period. A daily exposure action value of $2.5 \mathrm{~m} / \mathrm{s}^{2}$ is also specified for the same frequency weighting and reference period. For many pneumatically or hydraulically assisted tools, vibration emission values less than those fixed by the physical agents directive can be challenging to achieve in practice.

One of the most commonly adopted measures for lowering human exposure to hand-arm vibration is the use of vibration isolating, or antivibration, gloves. Antivibration gloves are attractive because they are applicable to existing tools, can be moved with the operator from one tool to the next, and can be less expensive than the purchase of tools characterised by lower vibration emissions. The vibration isolation is largely determined by the material properties and thickness of the cushioning materials interposed between the vibrating surface and the human hand. The identification of appropriate cushioning materials is therefore a key aspect of the exposure-reduction problem.

A promising new class of material which may prove useful in the glove cushioning application is that of auxetic materials. An auxetic material is defined as one having a negative Poisson's ratio, thus a material whose lateral extent will expand, rather than contract, under uniaxial tension. The term "auxetic" was first defined by Evans and co-workers at the University of Exeter ${ }^{(3)}$, and it is one of the definitions entered in the mainstream for these specific materials. Since 1987, when isotropic auxetic foam was first manufactured ${ }^{(4)}$, there has been much interest in developing negative Poisson's ratio materials for use in several engineering fields. The negative Poisson's ratio behaviour does not contradict the classical theory of elasticity. A homogeneous, isotropic, thermodynamically correct 3D solid has a potential Poisson's ratio range between -1.0 and 0.5 , while the magnitude of the Poisson's ratio can be even larger in the case of anisotropic solids ${ }^{(3)}$. A negative Poisson's ratio coefficient for a material can lead to increased indentation resistance ${ }^{(5)}$, enhanced bending stiffness in structural elements, increased shear resistance ${ }^{(6)}$ in structural elements, optimal passive tuning of structural vibration ${ }^{(7)}$ and enhanced dielectric properties for microwave absorbers ${ }^{(8)}$. The dynamic behaviour of auxetic materials has been investigated by several authors ${ }^{(9,10,11,12)}$. The possible use of auxetic materials for viscoelastic damping applications has been examined in ${ }^{(13)}$, where a biphasic 
auxetic composite showed a loss tangent exceeding the lower Voigt limit, and close to the Hashin upper bound. In recent papers ${ }^{(14,15)}$ increases in loss factor and storage moduli have been shown for auxetic PU foams manufactured using a modified route from the classical production layout. The unusual deformation mechanisms of auxetic foams have been also recently proposed for displacement control via magnetic fields in foam composite with one phase made of magnetorheological fluid ${ }^{(16)}$. One possible application of auxetic foams in ergonomics and human factors is that of special seat cushions ${ }^{(17)}$, where negative Poisson's ratio foams can be used to reduce interface pressures, thus reducing discomfort and possibly preventing pressure sores.

The testing of antivibration glove cushioning materials has been the subject of several research studies ${ }^{(19,20)}$ which have lead to the establishment ISO standard 13753:1999 Mechanical vibration and shock - Hand-arm vibration - Method for measuring the vibration transmissibility of resilient materials when loaded by the hand-arm system ${ }^{(21)}$, which defines a method for measuring the mechanical transmissibility of glove cushioning materials. As stated in ISO 13753:1999, "The method determines the impedance of the material when loaded by a mass providing a compression force equivalent to that found when the material is gripped by the hand. This is done by measuring the transfer function of the mass-loaded material at all the required frequencies. The vibration transmission when loaded by the hand is computed using standard values of hand-arm impedance and the measured values of the material impedance. The impedances used in this International Standard are for the palm of the hand when gripping a circular handle." ISO 13753:1999 provides a useful, and relatively simple, method for evaluating the isolation properties of resilient materials for antivibration gloves or other products which attempt to reduce the transmission of vibration to the human hand-arm system.

This paper describes a research study in which the possible antivibration properties of auxetic polyurethane foam have been evaluated. The research has been performed in light of the possibility of fabricating antivibration gloves from auxetic polyurethane foam, therefore the ISO 13753 method of defining material transmissibility has been adopted. In order to evaluate the performance of the auxetic foams that were defined and produced, comparisons are made with respect to a conventional polyurethane foam sample.

\section{FOAM SAMPLE MATERIAL PROPERTIES}

The specimens that were tested were produced from conventional grey opencells polyurethane foam with $30-35$ pores/inch and $0.0027 \mathrm{~g} / \mathrm{cm}^{3}$ density, 
supplied by the McMaster-Carr Co. of Chicago Illinois. The manufacturing process followed a procedure outlined in ${ }^{(15)}$. An aluminium mould consisting of cylinders backed by pistons was used to impose concurrent axial and radial compression on the parent foam samples. The compression ratio value ranged from 3 to 6 along the two manufacturing directions, followed by heating until the softening temperature was reached. The base foam was supplied in squared blocks of $600 \mathrm{~mm}$ side by $50 \mathrm{~mm}$ thickness. Cylindrical samples were obtained using a sharp-edge tube which was penetrated into the block of conventional foam. These samples were then inserted inside lubricated tubes, using a conical inlet to avoid creases. The tubes containing the parent foam were then positioned in the mould frame and a piston pushed inside it. The locking of the piston in the desired position followed compression. The whole operation was then repeated for the other specimens. The mould was then put into an industrial oven, and two heating profiles ${ }^{(15)}$ were used. The heating process was followed by room temperature cooling, which lasted two hours on average. After this operation, the extracted specimens were gently tensioned in order to relax the external surface. Following a procedure outlined in $^{(15)}$, the general designation of the samples was defined by an "XY" code, where " $X$ " was the position of the specimen in the mould and " $Y$ " was the progressive letter assigned to every specimen. Samples with higher uniformity of mechanical properties belonged to the specimens C and L (parameters shown in Table 1). For comparison, specimens with no imposed radial compression were also tested. These latter manufacturing parameters were labelled as "M" specimens. The specimens with no imposed radial load were produced as a control sample, to check against the possibility that measured changes in mechanical and transmissibility properties of the auxetic foams were due to the increase in foam density.

In a manner similar to the procedure outlined in ${ }^{(14,15)}$, Poisson's ratio measurements were based on image data acquired with a SONY DCR-TRV50E digital camera and processed with the MATLAB v6.1 software. The images were acquired

Table 1. Compression ratios used for the different auxetic and iso-density foams

\begin{tabular}{|l|c|c|c|}
\hline \multirow{2}{*}{ Sample series } & \multicolumn{3}{|c|}{ Compression ratio (\%) } \\
\cline { 2 - 4 } & Radial & Axial & Volumetric \\
\hline 3C & 36.7 & 46.9 & 78.7 \\
\hline $5 \mathrm{C}$ & 36.7 & 29.2 & 71.6 \\
\hline 2L & 62.0 & 50.0 & 92.8 \\
\hline 5M & 0 & 68.8 & 68.8 \\
\hline
\end{tabular}


during quasi-static tests performed using an MTS 858 servo-hydraulic machine. The Poisson ratio measures were carried out using specimens which were cut to $40 \mathrm{~mm}$ in length and glued to the end clamp of the machine with Loctite ${ }^{\circledR}$ adhesive, then stretched until breaking or ungluing at $0.1 \mathrm{~mm} / \mathrm{s}\left(\mathrm{e}=0.0025 \mathrm{~s}^{-1}\right)$. A calibration photo was taken for each test in order to convert the units of measurement from pixels to millimetres. A ruler was placed at a set distance from the camera iris, and the conversion factor was determined geometrically using the known distance of the ruler from the camera and the nominal focal length of the camera lens. After calibration, a set of photos was acquired to measure longitudinal and transverse strain during testing. This operation was performed using a MATLAB routine which calculated ten values of diameter and ten values of length along the specimen from every photograph, providing as output both the mean value and the standard deviation. To avoid end effects during the measurements, the extreme thirds of the dark-grey specimens were painted white in order to consider only the central third in the calculation of the lengths.

The MTS 858 servo-hydraulic system used for the materials properties testing included a cross-head-mounted actuator, a hydraulic power unit and a test controller with two channels of control (force and displacement). The load unit is fatigue-rated at $25 \mathrm{kN}$, and can be operated at frequencies up to $30 \mathrm{~Hz}$, with an integral crosshead-mounted linear actuator with an attached manifold. The MTS machine was used to perform tensile test (Poisson's ratio measures) and quasi-static compression tests on specimens $5 \mathrm{C}, 3 \mathrm{C}, 2 \mathrm{~L}, 5 \mathrm{M}$ and parent foam samples.

Quasi-static compression tests were carried out before the Poisson's ratio testing. A gap of $40 \mathrm{~mm}$ was left between the clamp ends of the machine, and samples cut to $40 \mathrm{~mm}$ in length were placed between them, lubricating the contact surfaces to minimize friction. The speed of the head was $0.01 \mathrm{mms}^{-1}$, indicating a strain-rate of $0.0025 \mathrm{~s}^{-1}$, with maximum compressive strain reached at $75 \%$. Data were acquired in displacement-crossing mode, acquiring displacement and force every $0.1 \mathrm{~mm}$. From the knowledge of the initial sizes of the specimens, it was possible to derive a strain-stress curve for every case. All tests were carried out at room temperature of $20^{\circ} \mathrm{C}$. No control of humidity was performed during the tests.

\section{ISO 13753 TRANSMISSIBILITY TESTS}

ISO 13753:1999 requires the measurement of the transfer function of the resilient material across the frequency range from 10 to $500 \mathrm{~Hz}$ while loaded 
by a steel cylinder which has a radius of $45 \mathrm{~mm}$ and a total mass of $2.5 \mathrm{~kg}$. The standard prescribes a material sample which is flat and of constant thickness, and which occupies the $45 \mathrm{~mm}$ radius area between the vibrating surface and the loading mass. The standard states that "The shaker may be driven by a wide-band random signal or a sinusoidal signal". For wide-band random signals, the standard specifies that "The power spectral density should be constant within +/- $10 \%$ between $10 \mathrm{~Hz}$ and $500 \mathrm{~Hz}$ with a magnitude of at least 2.5 x $10^{-2}\left(\mathrm{~m} / \mathrm{s}^{2}\right)^{2} / \mathrm{Hz}$.", while for sinusoidal excitation an input magnitude of at least $1 \mathrm{~m} / \mathrm{s}^{2}$ is required. For each individual band or frequency, the material impedance is calculated by means of the expression

$$
Z m=\frac{j \omega m}{\frac{A_{1}}{A_{2}}-1}
$$

where $A_{1}$ is the complex amplitude of the input excitation and $A_{2}$ is the complex amplitude of the output excitation of the loading mass. The vibration transmission of the resilient material when loaded by the human hand is then estimated using standard values of hand-arm impedance which are provided in the standard, and the measured values of the material impedance from expression (1), as

$$
T=\frac{Z m}{Z h+Z m}
$$

In order to perform the ISO 13753:1999 measurements, circular samples were manufactured as shown in Figure 1. The samples presented a minimum overall diameter $D$ of $95 \mathrm{~mm}$, with the inner diameter $d$ equal to the one obtained from the aluminium mould. The thickness of each sample was $10 \mathrm{~mm}$. Samples were placed between the $2.5 \mathrm{~kg}$ loading mass and the base board, which had overall dimensions of $200 \mathrm{~mm}$ x $200 \mathrm{~mm}$ x $12 \mathrm{~mm}$ and which had a total mass of $3.74 \mathrm{~kg}$. The baseboard was connected to a rigid frame by bungee cords and springs having a total stiffness of $0.36 \mathrm{Nmm}^{-1}$. The excitation to the baseboard was provided by a V406(SN 20333-2) electrodynamic shaker and accompanying power amplifier. A steel stinger rod with a $1 \mathrm{~mm}$ cross section was fixed to the baseboard with a screw-bolt system. Care was taken to align the stinger to the baseboard so as to guarantee a purely vertical motion of the baseboard. Two PCB Model 33C604 accelerometers were fixed to the loading mass and to the top of the baseboard using wax. The accelerometers (serial numbers 14582 and 14590) had calibration factors of $96.937 \mathrm{~ms}^{2} \bullet$ Volt and $94.966 \mathrm{~ms}^{2}$ -Volt respectively, and were amplified by a PCB Piezotronics Model 483A (SN 


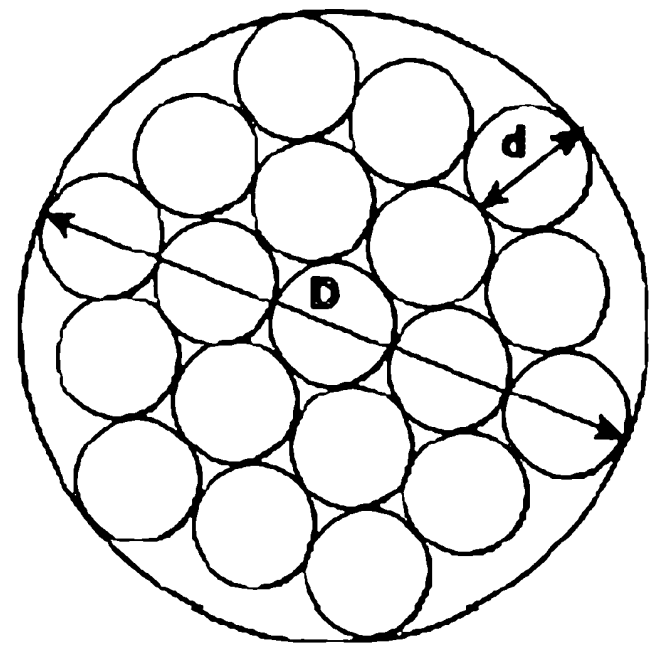

Figure 1. Diagram of sample geometry for the transmissibility tests

1355) amplifier. Figure 2 presents a schematic diagram of the laboratory test facility that was used. A DSP Siglab ${ }^{(22)}$ software based on MATLAB 6.5 was used to drive the shaker and to perform the Discrete Fourier Transform (DFT) of the signals from the accelerometers. The shaker was driven using a random white noise signal with energy in the frequency range from 1 to $500 \mathrm{~Hz}$, and r.m.s. voltage amplitude of $1.4 \mathrm{~V}$, and r.m.s. acceleration at the mass head of $1275 \mathrm{~ms}^{-2}$. The transmissibility of the material samples was calculated over the frequency range from 1 to $500 \mathrm{~Hz}$ using 1024 spectral lines and a $50 \%$ window overlap, with overload block rejection. Hanning windows were used for all data acquisitions.

\section{RESULTS AND DISCUSSION}

Figure 3 presents a comparison between the Poisson's ratio of the conventional foam and those of auxetic specimens 3C, 5C, 2L and 5M. The Poisson's ratio values are all strain dependent, tending towards near-zero, or positive values, for large tensile strains. The foam of specimen $3 \mathrm{C}$ had its highest magnitude of Poisson's ratio for a tensile strain of approximately 90\%. For specimen 5C, the minimum Poisson's ratio values occurred at low tensile strains, with NPR average values of -0.22 . Beyond a tensile strain of $50 \%$, the Poisson's ratio of specimen $5 \mathrm{C}$ decreased with the applied strain, becoming positive at $100 \%$ 


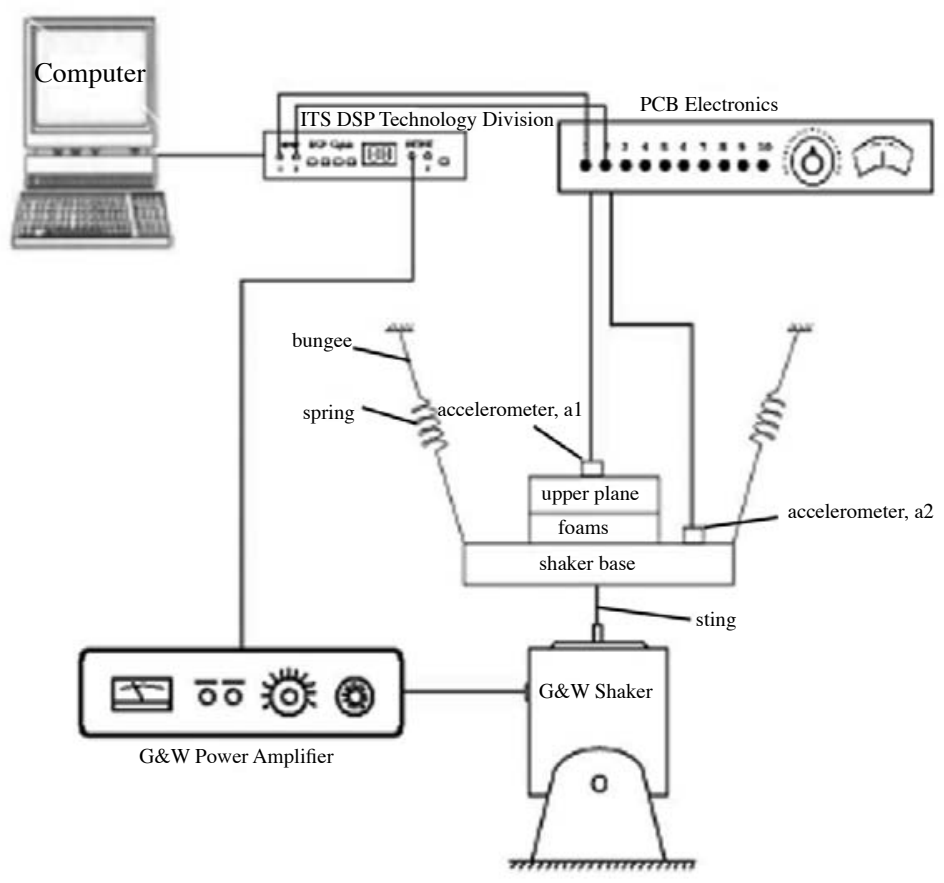

Figure 2. Schematic layout of the transmissibility test rig

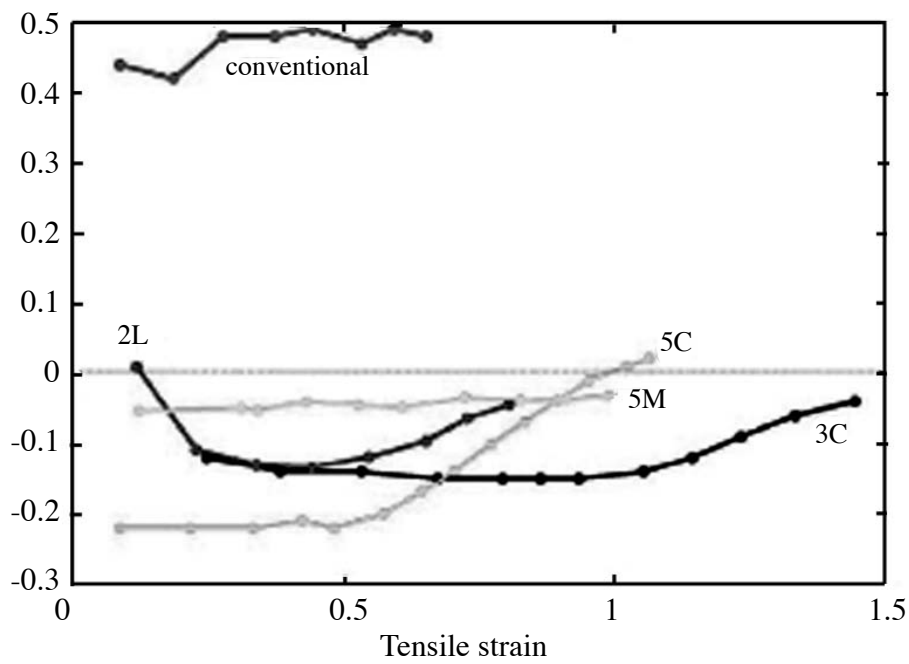

Figure 3. Comparison of the Poisson's ratio against tensile strains for the different types of foam 
elongation. Specimen $2 \mathrm{~L}$ presented a negative Poisson's ratio behaviour, with lower sensitivity to tensile strain, becoming near zero for tensile strains above $90 \%$. The iso-density foam $5 \mathrm{M}$ presented negative, albeit small, Poisson's ratio values. Theoretically, a zero radial compression should not be capable of generating a negative Poisson's ratio ${ }^{(4)}$ in a foam. In practice, during the manufacturing process the axial compression applied to the sample can be sufficient to push the foam specimen against the cylindrical walls of the mould, providing a radial compression of the specimen. It can be noted, however, that the values of the NPR are confined within the range -0.04 and -0.05 . Figure 4 presents a comparison between the compressive stress-strain curves of the auxetic foams and that of the conventional parent foam. The auxetic foams do not show the classical stress-strain compressive pattern of conventional foams ${ }^{(15)}$. Instead, the characteristic is exponential, with compressive stresses around densification (above 50\% strain) being almost two orders of magnitude higher compared to that of the conventional parent foam. The iso-density foam, on the other hand (Figure 5), was characterised by higher compressive stresses than the conventional foam, but its stiffness was lower than that of the auxetic foam (5C) for compressive strains up to $23 \%$. After this value, the stiffness of foam $5 \mathrm{M}$ was significantly higher than the $5 \mathrm{C}$ counterpart, with a compressive stress of $140 \mathrm{kPa}$ at $50 \%$ compared to the $60 \mathrm{kPa}$ of the more auxetic foam at the same compressive strain level.

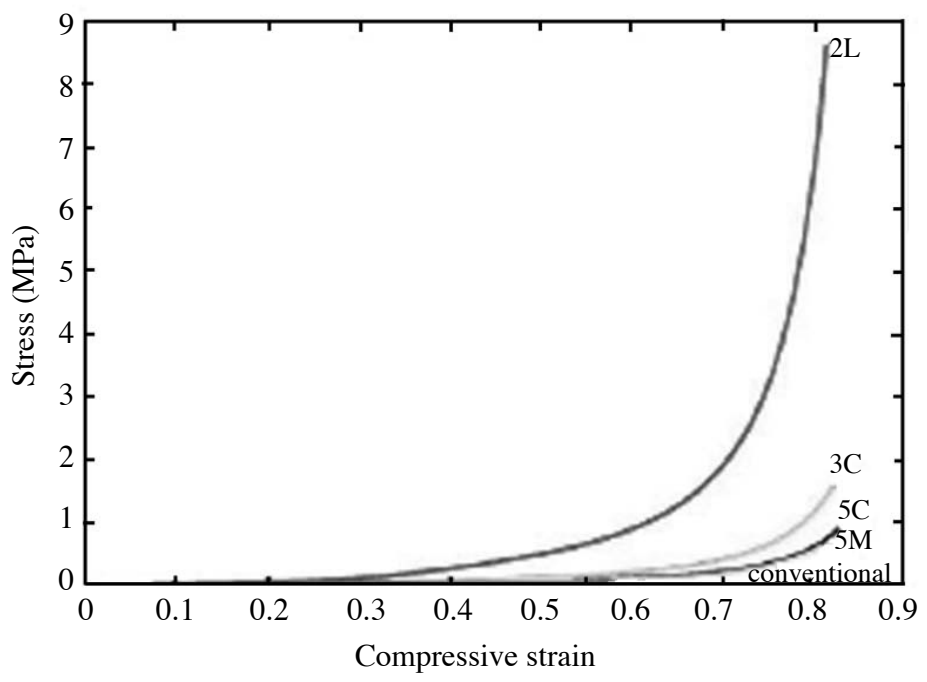

Figure 4. Compressive stress-strain curves for the different types of foams 


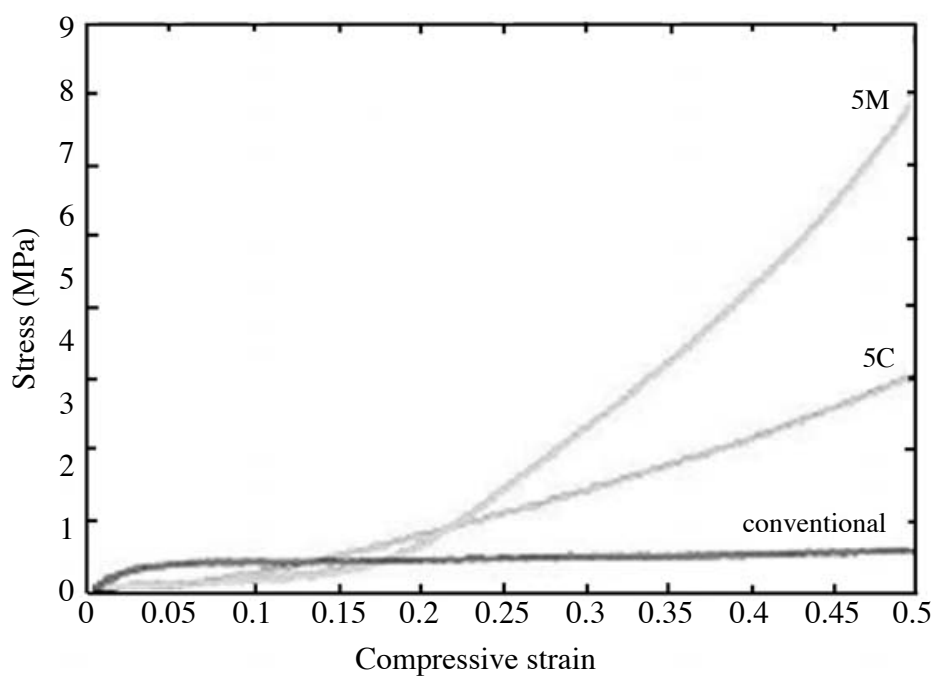

Figure 5. Comparison of compressive stress-strain curves for conventional, types 5C and $5 \mathrm{M}$ foams up to $50 \%$ of strain

Figure 6 presents the ISO 13753 transmissibility of the foams. Within the frequency range from 10 to $31.5 \mathrm{~Hz}$ it can be observed that all the transmissibility values were found to be greater than 1 , meaning that all the foams increased the magnitude of the transmitted vibration. This result is not unusual for thin, resilient, materials $^{(18)}$ in the hand-arm application. Over the 10 to $31.5 \mathrm{~Hz}$ frequency range the specimen $\mathrm{C}$ auxetic foam produced the highest transmissibility values, while foam specimen $2 \mathrm{~L}$ provided the lowest. For frequencies greater than $31.5 \mathrm{~Hz}$, all the foam specimens provided attenuation against vibration. It is worthy of note that although the auxetic L specimen provided better vibration isolation performance below $31.5 \mathrm{~Hz}$, the attenuation was lower compared to the conventional foam at higher frequencies. At the higher frequencies, the lowest transmissibility values were achieved by the lower manufacturing volumetric ratio auxetic foams (specimens C). For Example, at $100 \mathrm{~Hz}$ the conventional foam provided a transmissibility value of 0.9 , while the auxetic $5 \mathrm{C}$ foam provided a value of 0.8 . From this trend, it might be presumed that decreases in foam volumetric ratio produce changes in the transmissibility curve of the auxetic foam which move the curve in the direction of that of the corresponding conventional foam. If further research were to confirm this hypothesis, it would be possible to identify an optimised manufacturing volumetric ratio starting from a target transmissibility specification. 


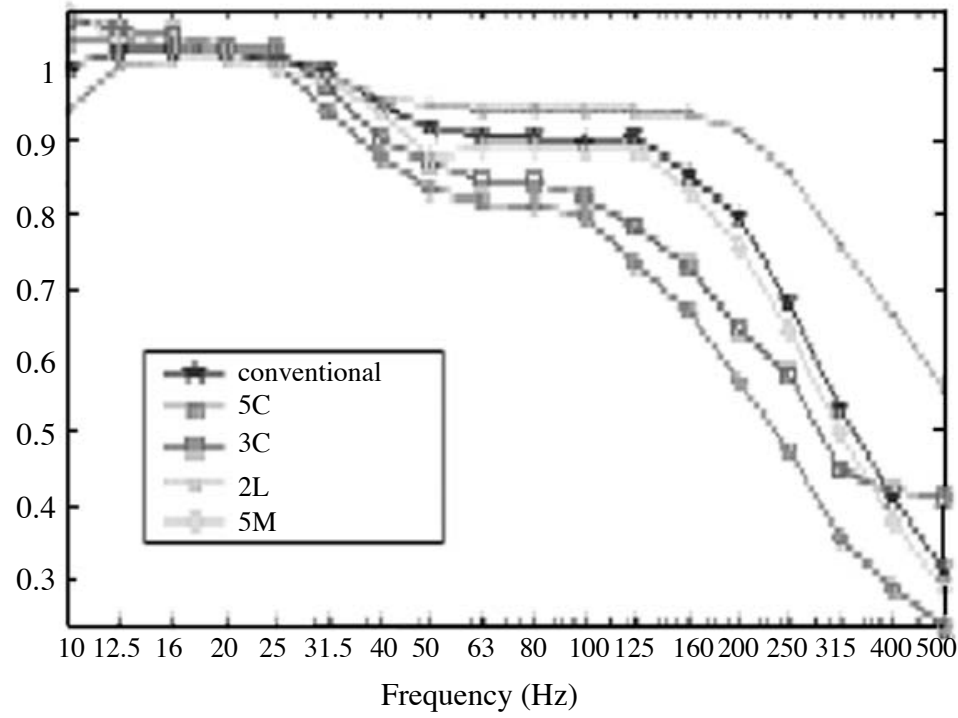

Figure 6. ISO 13753 transmissibility ratios against frequency for the different types of foams

In the ISO 13753 transmissibility tests described above, the application of the loading mass on the foam samples lead to an indentation corresponding to a compressive strain of $10 \%$. Table 2 presents the compressive stresses corresponding to the indentation strain for the different foam specimens that were tested. Foam specimen $2 \mathrm{~L}$ provided the highest stiffness, while foam specimen $5 \mathrm{M}$ provides the lowest. Figure 7 presents the transmissibility characteristics of the foams by means of a combined frequency-compressive stress diagram. It can be observed that the foam specimen with the highest stiffness (2L) provided the lowest transmissibility values at frequencies below $31.5 \mathrm{~Hz}$. This result may be due to the stiffness-driven behaviour of the system at low frequencies.

Table 2. Comparison of stress levels at $10 \%$ compressive strain for the different foams

\begin{tabular}{|l|c|c|c|c|c|}
\hline & Conventional & 5C & 3C & 2L & 5M \\
\hline Stress $(\mathrm{KPa})$ at $10 \%$ strain & 14 & 9 & 10 & 20 & 4 \\
\hline
\end{tabular}




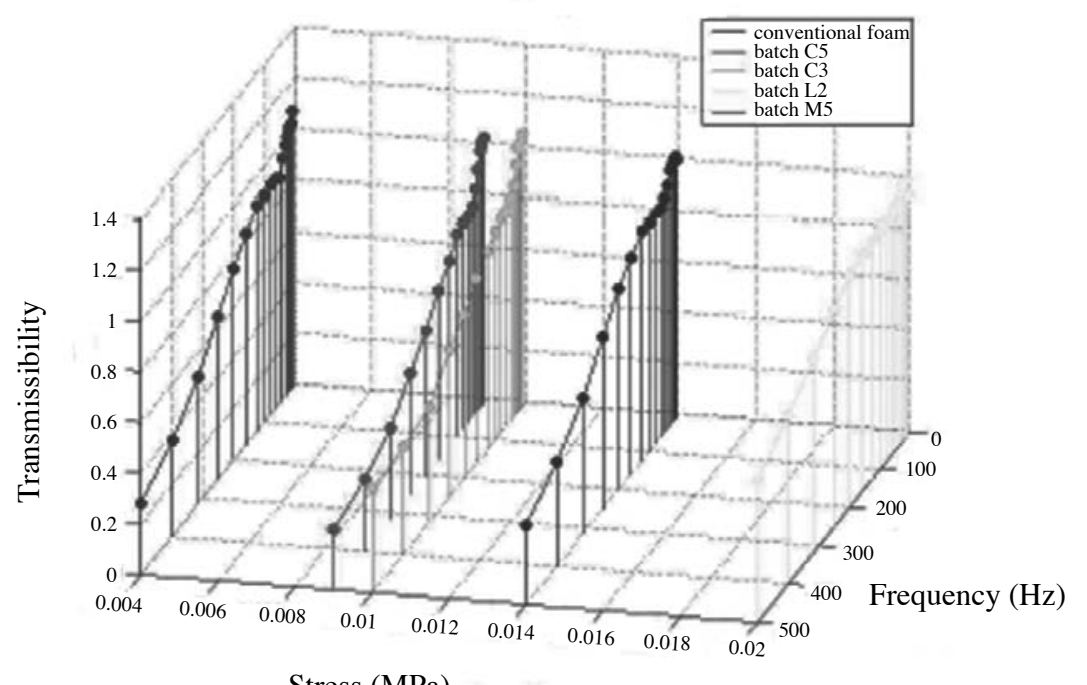

Stress (MPa)

Figure 7. ISO 13753 transmissibility ratios versus frequency and compressive stresses at $\mathbf{1 0 \%}$ compressive strain for the different types of foams

Inspection of Figures 6 and 7 suggests that none of the foams which were developed and tested in the course of the current study achieved ISO 13753:1999 transmissibilities as low as the best materials identified by Koton et al. ${ }^{(18)}$. As an example, the five best materials (not all of which were polymeric) identified by Koton et al. had transmissibilities in the range from 0.84 to 0.96 at $20 \mathrm{~Hz}$, compared with values of approximately 1.0 for the foams developed in the current study. Further, the five best materials of Koton et al. had transmissibility values in the range from 0.37 to 0.56 at $100 \mathrm{~Hz}$, compared with values from 0.79 to 0.94 for the foams developed in the current study. The set of manufacturing parameters utilised for the foams of the current study were therefore not strictly optimal from the point of view of vibration transmissibility.

However, the performance of materials used in antivibration gloves should also be quantified in terms of the indentation characteristics of the resilient

Table 3. Density values for the different types of foam

\begin{tabular}{|l|c|c|c|c|c|}
\hline & Conventional & 5C & 3C & 2L & 5M \\
\hline Density $\mathrm{g} / \mathrm{cm}^{3}$ & 0.0272 & 0.091 & 0.112 & 0.254 & 0.075 \\
\hline
\end{tabular}


materials. It can be hypothesised that a better distribution of the pressure at the interface between the glove and the human hand, meaning lower peak interface pressures, will result less damaging to skin tissue and to blood capillaries, even if associated with higher overall levels of vibration transmitted to the hand. This possibility, worthy of further research, suggests an important advantage of auxetic materials, that of redistributing laterally the pressure field due to the negative Poisson ratio. As a demonstration of this potentially beneficial effect, a finite element model was developed using the commercial FE code ANSYS 8.0 ${ }^{(23)}$ which consisted of a $2 \mathrm{D}$ foam block with dimensions of $200 \mathrm{~mm} \times 200 \mathrm{~mm}$. The foam block was subjected to an indentation of $1 \%$ of compressive strain over one quarter of the block side. The model was prepared using 2D, 4 node, isoparametric PLANE42 elements with Lagrange interpolation functions in plain strain. Due to the small strain involved, linear elastic parameters were used for the material characterisation, i.e., Young's modulus and Poisson's ratio. The model was axisymmetric, had 1470 elements, and was loaded in the central portion. Two analysis cases were considered: the conventional base foam (Young's modulus $380 \mathrm{kPa}$, Poisson's ratio 0.4), and the auxetic foam type 5C (Young's modulus $115 \mathrm{kPa}$, Poisson's ratio -0.23 ). Figure 8 presents the total sum of deformation for the conventional foam case (Figure 8(a)) and for the negative Poisson's ratio one (Figure 8(b)). The conventional foam presents the lower deformation fields at the top right edge and lower bottom (towards the axis of symmetry) of the block, while two orders of magnitude higher of deformation are recorded in the quarter bottom right of the block. The auxetic foam, instead, tends to enfold around the indentation zone, providing a wrapping effect not present in the conventional cellular material. The orders of magnitude of deformation for the two types of foams are substantially equivalent, with a slight increase of

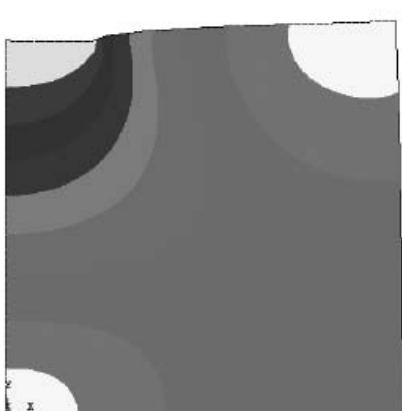

(a)

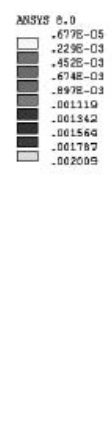

(b)

Figure 8. Total sum of deformations for the FE indentation model: (a) conventional foam; (b) auxetic 5C 


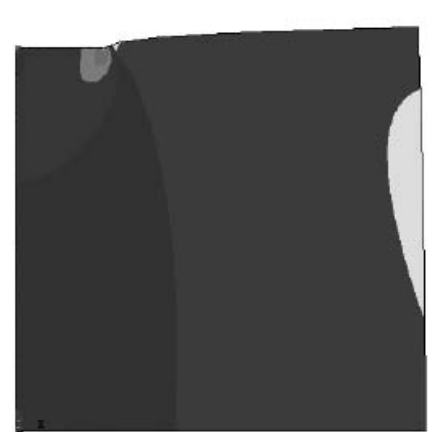

(a)

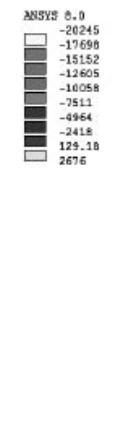

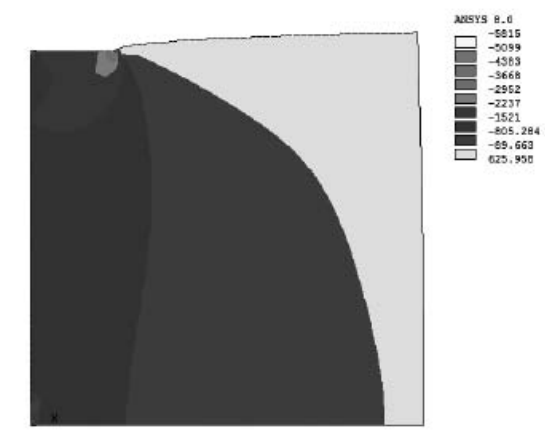

(b)

Figure 9. Normal stresses (y-direction) for the FE indentation model: (a) conventional foam; (b) auxetic 5C

$6 \%$ on average for the auxetic solid. The normal stress distributions (along the $y$-direction of the foam blocks) for the conventional (Figure 9a) and auxetic foam (Figure 9b) show a significant drop in terms of average normal stresses around the indentation area for the auxetic cellular solid, with an average value of $1.52 \mathrm{kPa}$ compared to $4.96 \mathrm{kPa}$ of the conventional foam. This decrease of a factor 3.2 is also present an average on the whole foam block. These results are consistent with the ones outlined $\mathrm{in}^{(17)}$. It is worthy of note that $72 \%$ of the auxetic foam block presents compressive normal stresses, while only $41 \%$ of the conventional foam records compressive stress fields, and the rest of the block foam provides a tensile stress relieve.

\section{CONCLUSIONS}

In this study the static and dynamic characteristics of conventional open cell polyurethane (PU), of auxetic and of iso-density foams have been analysed for possible antivibration glove applications. For the auxetic foams developed during the current study, the results suggested similar behaviour to that previously reported in the research literature for auxetic foams, with significant increases in stiffness during compressive loading, and a significant dependence of the Poisson's ratio on the applied strain. The transmissibility tests, performed in accordance with the ISO 13753 procedure for resilient materials, suggested a strong dependence of the transmissibility on the foam manufacturing parameters. Within the frequency range from 10 to $31.5 \mathrm{~Hz}$ the transmissibility was found to be greater than 1 , while it was less than 1 at all frequencies greater than $31.5 \mathrm{~Hz}$. Comparison of the transmissibility results 
to data available in the research literature for glove materials suggested that the foams tested in the current study provided values similar to the mean values for 80 resilient materials tested by Koton et. al., but were significantly higher than the five best materials (not all polymeric) identified by the same researchers. In this study it has been suggested, however, that the resilient behaviour of glove isolation materials should also be evaluated in terms of the indentation characteristics. It can be hypothesised that a better distribution of the pressure at the interface between the glove and the human hand will result less damaging to skin tissue and to blood capillaries, even if associated with higher overall levels of vibration transmitted to the hand. A simple, linear elastic, Finite Element simulation was performed, and the indentation results suggest that auxetic foams offer a significant decrease in compressive stresses with respect to conventional PU foams.

\section{ACKNOWLEDGEMENTS}

This work has been partially supported by the UK Engineering and Physical Science Research Council grant GR/97303.

\section{REFERENCES}

1. European Union Directive 2002/44/EC of the European Parliament and of the Council of 25 June 2002 on the minimum health and safety requirements regarding the exposure of workers to the risks arising from physical agents (vibration) (sixteenth individual Directive within the meaning of Article 16(1) of Directive 89/391/EEC), Official Journal of the European Communities, L 177, 13-19.

2. International Standard ISO 5349-1 2001, Mechanical Vibration - Measurement and assessment of human exposure to hand-transmitted vibration - Part 1: General guidelines, International Organization for Standardization, Geneva.

3. Evans, K. E. 1991. Endeavour, New Series, 15(4), 170.

4. Lakes R. S., 1987. Science, 253, 1038.

5. Lakes R. S. and Elms K., 1993. Journal of Composite Materials, 27, 1193.

6. Lakes R. S., 1993. ASME Journal of Mechanical Design 115, 696.

7. Scarpa F. and Tomlinson G., 2000. Journal of Sound and Vibration 230(1), 45.

8. Smith F. C., Scarpa F. and Chambers, B., 2000. IEEE Microwave and Guided Wave Letters 10(11), 451.

9. A.W. Lipsett, A. I. Beltzer, 1988, J. Acoust. Soc. Am., 84, 2179. 
10. C. P. Chen, R. S. Lakes, 1989. Cellular Polymers, 8, 343.

11. B. Howell, P. Prendergast, L. Hansen, 1991. 'Acoustic behaviour of negative Poisson's ratio materials', DTRC-SME-91/01, David Taylor Research Centre, Annapolis, MD.

12. E. O. Martz, R. S. Lakes, J. B. Park, 1996. Cellular Polymers, 15(5), 349.

13. C. P. Chen, R. S. Lakes, 1996. J. Engineering Materials and Technology, 118, 285.

14. F. Scarpa, L. G. Ciffo, J. R. Yates, 2004. Smart Materials and Structures, 13, 49.

15. Scarpa, F., Pastorino, P., Garelli, A., Patsias, S. and Ruzzene, M., 2005. Physica Status Solidi B, 242(3), 681.

16. Bullough, W. A. and Scarpa, F., 2005. International Journal of Modern Physics $B, 19(7-9), 1655$.

17. Wang Y. C. and Lakes, R., 2002. International Journal of Solids and Structures, 39(18), 4825.

18. Koton, J., Kowalski, P. and Szopa, J. 1998. Proceedings of the 8th International Conference on Hand-Arm Vibration, 9-12 June, Umeå, Sweden, 329-335.

19. Cronjäger, L., Zeglam-Verch, I. And Gillmeister, F. 1992. Proceedings of the 6th International Conference on Hand-Arm Vibration, Bonn, Germany, 19-22 May, 747-754.

20. Smagowska, B. and Liwkowicz, J. 1992 Proceedings of the 6th International Conference on Hand-Arm Vibration, Bonn, Germany, 19-22 May, 755-764.

21. International Organisation for Standardisation 1999, ISO 13753:1999 Mechanical vibration and shock - Hand-arm vibration-Method for measuring the vibration transmissibility of resilient materials when loaded by the hand-arm system (Geneva: International Organisation for Standardization).

22. SIGLAB 6.1 Users'Manual, 2001, Spectral Dynamics Inc., San Jose, CA.

23. ANSYS 8.0 User's Manual 2004, Ansys Inc, Canongsburg, PA. 